\title{
Rising Prevalence of Comorbid Alcohol and Opioid Use Disorders in Adolescents and Young Adults in the United States
}

\author{
Elissa R. Weitzman, ScD, MSc ${ }^{1,2,3}$ and Mei-Sing Ong, PhD
}

'Department of Pediatrics, Harvard Medical School, Boston, MA, USA; 2Division of Adolescent/Young Adult Medicine, Boston Children's Hospital, Boston, MA, USA; ${ }^{3}$ Computational Health Informatics Program, Boston Children's Hospital, Boston, MA, USA; ${ }^{4}$ Department of Population Medicine, Harvard Medical School \& Harvard Pilgrim Healthcare Institute, Boston, MA, USA.

J Gen Intern Med 34(10):1987-9

DOI: $10.1007 / \mathrm{s} 11606-019-05068-6$

(c) Society of General Internal Medicine 2019

\section{INTRODUCTION}

National surveys reveal changing patterns of alcohol use among adolescents and young adults (AYA) over the past decade, toward lower use overall, but sharply rising levels of high intensity use. ${ }^{1}$ During this time, rates of opioid misuse and mortality have risen dramatically among AYA. ${ }^{2}$ Because both alcohol and opioids are central nervous system depressants, concomitant use of alcohol and opioids poses heightened risk of respiratory depression and overdose. Alcohol is involved in over $20 \%$ of opioid-related overdose deaths, ${ }^{3}$ and co-occurring alcohol use disorder (AUD) significantly increases the risk of all-cause mortality among opioid use disorder (OUD) patients. ${ }^{4}$ Furthermore, dual diagnosis of AUD and OUD complicates treatment for either condition alone. ${ }^{5}$ We examined the magnitude and trends of co-occurring diagnoses of AUD and OUD among AYA nationwide to quantify problems within routinely collected healthcare administrative claims data.

\section{METHODS}

We analyzed deidentified claims data from a nationwide US health insurance plan. The Boston Children's Hospital Institutional Review Board approved the study. Subjects were AYA aged 10 to 26 years enrolled in the healthcare plan between years 2008 and 2015. Cases of AUD were identified from diagnoses of alcohol dependence (ICD-9303) and non-dependent alcohol abuse (ICD-9305.0); cases of OUD were identified from diagnoses of opioid dependence (ICD-9304.0, 304.7) and nondependent opioid abuse (ICD-9305.5). Analyses were stratified by two age groups (ages 10-17 and 18-26 years). Statistical analyses were performed using $\mathrm{R}$ statistical language version 3.0.

\section{RESULTS}

Of 21,824,756 AYA enrolled in the healthcare plan, 148,210 (40.5\% female) and 50,144 (34.8\% female) satisfied the

Published online May 31, 2019 inclusion criteria for AUD and OUD, respectively. From 2008 to 2015, upward trends in AUD and OUD were observed when the population is considered as a whole and among AYA ages 18-26 years (Fig. 1). In both age groups, there was a rising prevalence of comorbid AUD and OUD (Fig. 2), most acutely among AYA aged 18-26 years: in 2015, 23.0\% $(n=$ $22,456)$ of those with AUD and $42.3 \%(n=12,208)$ of those with OUD were dually affected, compared with $8.5 \%(n=$ $14,447)$ of AUD and $28.6 \%(n=4167)$ of OUD patients in 2008. For AYA aged 10-17 years, AUD prevalence declined, while the prevalence of OUD remained unchanged. The prevalence of comorbid AUD and OUD among this group increased from 2008 to 2012 and plateaued thereafter; in 2015, 6.5\% $(n=2255)$ with AUD and $38.5 \%(n=379)$ with OUD were dually diagnosed.

\section{DISCUSSION}

Sizeable and increasing proportions of AYA have either alcohol or opioid use disorder, of which substantial percentages have co-occurring disorders, consistent with survey studies. ${ }^{6}$ Considerable attention has been paid to the challenges posed by opioid misuse among AYA in the USA; however, scant attention has been paid to the potential that alcohol use problems may compound these concerns and need simultaneous treatment. Despite declines in alcohol use among AYA overall, findings of rising prevalence of AUD show alignment with reports of rising levels of high-intensity use among older AYA - a complex group given the large minority with co-occurring OUD. Trends in comorbid AUD and OUD may have plateaued for AYA aged 10-17 years since 2012; however, the proportion of dually affected patients remained strikingly high. Research is needed to investigate the temporal relationship and predictors of comorbid AUD and OUD to drive effective interventions at the earliest possible moment in a young life.

\section{Limitations}

Analyses reflect commercially insured AYA and may not generalize to other populations. The prevalence of AUD and OUD and their co-occurrence are likely to be higher in uninsured populations. Behavioral health issues are often under- 


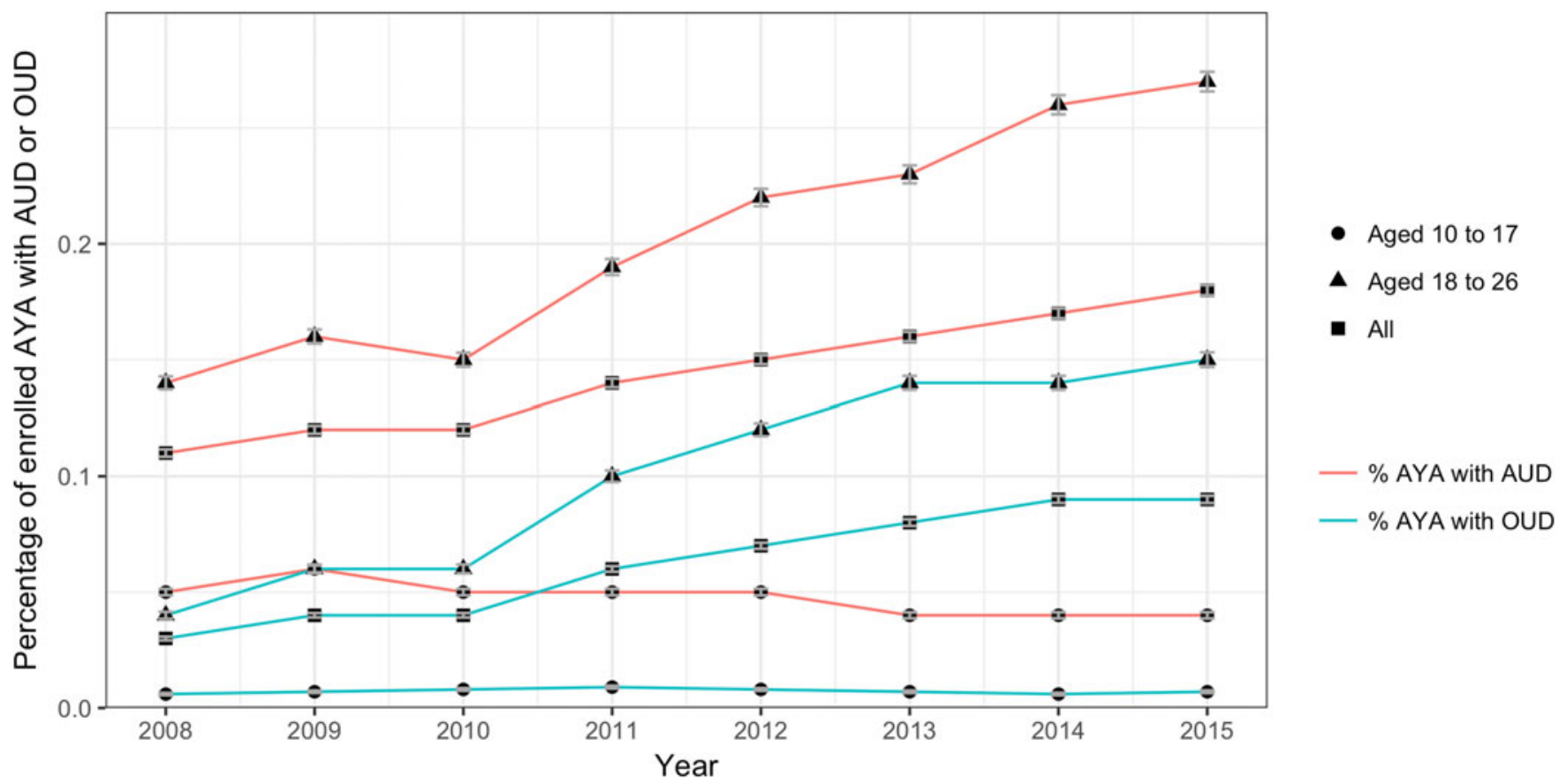

Figure 1 Percentage of adolescent and young adult insurance plan members ages 10-26 years with an alcohol or opioid use disorder over the 2008-2015 period in total and by age group. Red lines depict the percentage of adolescent and young adult insurance plan members ages 10-

26 years who have an alcohol use disorder in total $(\bullet)$ and for youth ages 10-17 years $(\Delta)$ and 18-26 years ( $\bullet)$. Green lines depict the percentage of adolescent and young adult insurance plan members ages 10-26 years with an opioid use disorder in total (•) and for youth ages 10-17 years $(\Delta)$ and 18-26 years $(\square)$.

coded in administrative claims; reporting biases may therefore yield underestimates of the problem, and the temporal relation between AUD and OUD is difficult to ascertain. Nevertheless, the availability of a large number of patients across facilities, geographical locations, and population demographics enables research into the burden of substance-related disorders at the

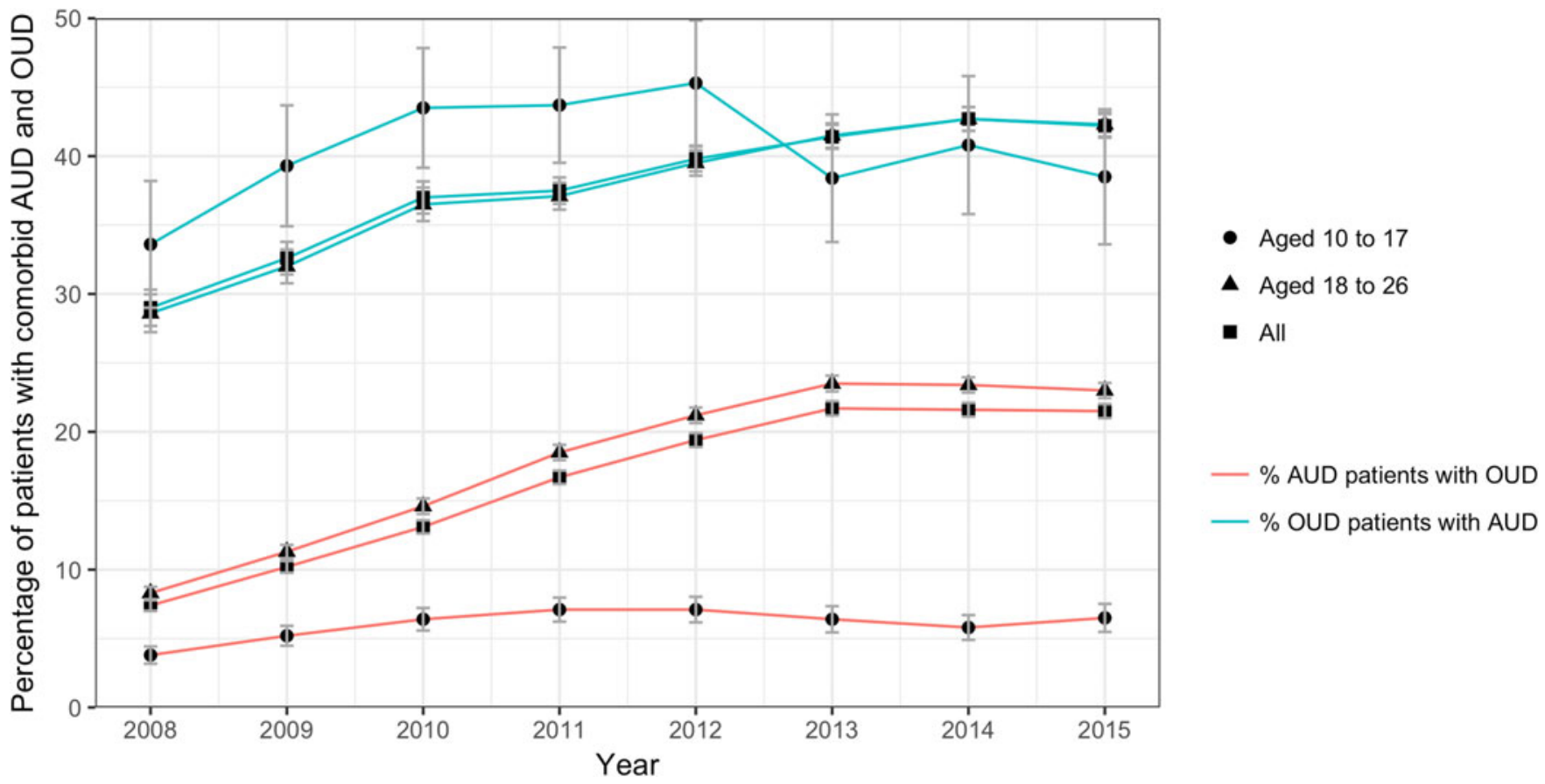

Figure 2 Percentage of adolescent and young adult insurance plan members ages 10-26 years with dual alcohol and opioid use disorders over the 2008-2015 period in total and by age group. Red lines depict the percentage of adolescent and young adult insurance plan members ages 10-26 years who have an alcohol use disorder and who also have an opioid use disorder in total $(\bullet)$ and for youth ages $10-17$ years $(\Delta)$ and $18-$ 26 years (๘). Error bars indicate $95 \%$ confidence intervals. Green lines depict the percentage of adolescent and young adult insurance plan members ages 10-26 years with an opioid use disorder who also have an alcohol use disorder in total $(\bullet)$ and for youth ages 10-17 years $(\Delta)$ and 18-26 years (ם). Error bars indicate $95 \%$ confidence intervals 
population-level. Furthermore, claims data are systematically collected during the course of medical care, thus limiting response and recall biases often encountered in survey-based studies.

\section{Conclusion}

Rising rates of alcohol and opioid use problems and their cooccurrence are evident among commercially insured AYA nationwide. Given the lifelong impact of substance use disorders, comprehensive longitudinal analyses are urgently needed to better understand these problems and to identify critical junctures for intervention.

Acknowledgments: We gratefully acknowledge Kara Magane for assisting with the identification of claims codes, reviewing and proof reading the manuscript, and preparing the article for submission.

Corresponding Author: Elissa R. Weitzman, $\mathrm{ScD}$, MSc; Department of Pediatrics Harvard Medical School, Boston, MA, USA (e-mail: Elissa.Weitzman@childrens.harvard.edu).

Funding This study was funded, in part, by the Maternal and Child Health Bureau (Title V, Social Security Act), Health Resources and Services Administration award \#T71MC00009.

\section{Compliance with Ethical Standards:}

Conflict of Interest: The authors declare they do not have a conflict of interest.
Disclaimer: The funding bodies were not involved in the design of the study or collection, analysis, and interpretation of data, nor in writing, review, or approval of the manuscript.

\section{REFERENCES}

1. Hingson RW, Zha W, White AM. Drinking beyond the binge threshold: predictors, consequences, and changes in the U.S. Am J Prev Med. 2017;52(6):717-727. https://doi.org/10.1016/j.amepre.2017.02.014.

2. Curtin SC, Tejada-Vera B, Warner M. Drug Overdose Deaths Among Adolescents Aged 15-19 in the United States: 1999-2015. Hyattsville; 2017. NCHS data brief, no 282.

3. Jones CM, Paulozzi LJ, Mack KA, Centers for Disease Control and Prevention (CDC) Alcohol involvement in opioid pain reliever and benzodiazepine drug abuse-related emergency department visits and drug-related deaths - United States, 2010. MMWR Morb Mortal Wkly Rep. 2014;63(40):881-885. https://doi.org/10.1098/rstb.2013.0595.

4. Bogdanowicz KM, Stewart R, Broadbent $\mathbf{M}$, et al. Double trouble: psychiatric comorbidity and opioid addiction-all-cause and cause-specific mortality. Drug Alcohol Depend. 2015;148:85-92. https://doi.org/10. 1016/j.drugalcdep.2014.12.025.

5. Witkiewitz K, Votaw VR, Vowles KE, Kranzler HR. Opioid misuse as a predictor of alcohol treatment outcomes in the COMBINE study: mediation by medication adherence. Alcohol Clin Exp Res. 2018;42(7):1249-1259. https://doi.org/10.1111/acer.13772.

6. McCabe SE, Veliz P, Patrick ME. High-intensity drinking and nonmedical use of prescription drugs: results from a National Survey of 12th grade students. Drug Alcohol Depend. 2017;178:372-379. https://doi.org/10. 1016/j.drugalcdep.2017.05.038.

Publisher's Note Springer Nature remains neutral with regard to jurisdictional claims in published maps and institutional affiliations. 